\title{
Susceptibilidad a azoles y anfotericina B de aislados de Candida spp. Experiencia de una red de salud universitaria, entre 2004 y 2010
}

\author{
Lorena Porte, Pilar León, Cynthia Gárate, Ana María Guzmán, Jaime Labarca y Patricia García
}

\section{Susceptibility to azoles and amphotericin B of isolates of Candida spp. Experience of a university health network, between 2004 and 2010}

Objective: To describe antifungal susceptibility testing surveillance (December 2004-September 2010) in Candida spp., for amphotericin B, fluconazole and voriconazole, at the Laboratorio de Microbiología, Pontificia Universidad Católica de Chile. Method: The study was performed utilizing E test and included yeasts from invasive origin and isolates in which antifungal susceptibility testing was asked for by the patient's physician. Results: The yeasts were mainly recovered from urine samples (n: 64), blood cultures (n: 51) and secretions (n: 24). Two hundred ninety three isolates were studied: C. albicans (38\%), C. glabrata (30\%), C. tropicalis (11\%), C. parapsilosis (10\%), C. krusei (4\%) and others (7\%). All Candida species were 100\% susceptible to amphotericin B, except C. krusei (1/12). Fluconazole's global susceptibility in C. albicans was $91.8 \%$, but $100 \%$ in isolates from blood cultures versus $76 \%$ in isolates from urine. C. tropicalis was $93.9 \%$ susceptible to fluconazole, C. parapsilosis, $90 \%$ and C. glabrata $30.3 \%$. C. krusei had no susceptible isolates to fluconazole. Voriconazole resistance was mainly present in C. glabrata (11.5\%). Conclusions: We recommend the study of antifungal susceptibility in isolates from invasive origin, selected urine strains and C. glabrata. Fluconazole remains effective in C. albicans from blood.

Key words: Candida, drug resistance, fluconazole, voriconazole, amphotericin B.

Palabras clave: Candida, resistencia antimicrobiana, fluconazol, voriconazol, anfotericina B.

\section{Introducción}

$\mathrm{D}$ esde la década de 1980 los hongos han emergido como importantes agentes de infecciones oportunistas en población inmunocomprometida ${ }^{1}$. Una de las principales etiologías de infecciones invasoras son las levaduras del género Candida. Candida albicans sigue siendo el hongo patógeno más común y ha mantenido un bajo nivel de resistencia antifúngica. Se ha descrito un aumento significativo de especies no-albicans, las que exhiben resistencia a azoles ${ }^{2}$. Por esta razón, actualmente se requiere conocer la susceptibilidad in vitro de los aislados con el fin de orientar y conseguir el éxito terapéutico.

La susceptibilidad antifúngica in vitro generalmente se cuantifica utilizando la concentración inhibitoria mínima $(\mathrm{CIM})^{3}$. Actualmente, el método de referencia para determinar la susceptibilidad antifúngica in vitro de Candida spp., es la dilución en caldo descrita por Clinical and Laboratory Standards Institute (CLSI) en su documento $\mathrm{M} 27-\mathrm{A}^{4}$. El suplemento M27-S3 ${ }^{5}$ de dicho documento presenta los puntos de corte correspondientes para fluconazol, itraconazol, voriconozol y equinocandinas. Esta metodología es difícil de realizar y de integrar a la rutina diaria en los laboratorios asistenciales. Los siste- mas comerciales de determinación de la susceptibilidad antifúngica in vitro como las tiras de $\mathrm{E}$ test $^{\circledR}\left(\right.$ bioMérieux $\left.^{\circledR}\right)$ presentan buena correlación categórica con el método de referencia y son una metodología alternativa validada para realizar este estudio de rutina ${ }^{6}$.

Nuestro laboratorio realiza estudio de susceptibilidad in vitro a antifúngicos en cepas del género Candida mediante método de $\mathrm{E}$ test ${ }^{\circledR}$ desde diciembre 2004. La indicación de realizar este estudio se limita a levaduras de muestras invasoras, cepas derivadas desde otros laboratorios o por solicitud expresa del médico tratante. El objetivo de este trabajo es reportar la susceptibilidad antifúngica in vitro de las principales levaduras estudiadas en nuestra institución durante un período de cinco años, con el fin de orientar al medico clínico en la elección de la terapia antifúngica empírica más apropiada.

\section{Material y Método}

El estudio realizado fue de corte retrospectivo y descriptivo. Se efectuó una búsqueda en el sistema computacional del laboratorio de todos los aislados de Candida spp., obtenidos a partir de muestras clínicas, a los que se
Pontificia Universidad Católica de Chile.

Departamento de Laboratorios Clínicos Laboratorio de Microbiología (LP, PL, CG, AMG, PG) Escuela de Medicina, Departamento de Medicina Interna (JL) Hospital Militar de Santiago Unidad de Microbiología, Laboratorio Clínico (LP)

Conflictos de interés: J. Labarca L. ha recibido financiamiento para investigación de Pfizer, MSD y Novartis. El resto de los autores declara no tener conflictos de interés.

Este proyecto no cuenta con financiamiento.

Recibido: 30 de junio de 2011 Aceptado: 9 de enero de 2012

Correspondencia a: Lorena Porte Torre nenapor@gmail.com 
les había realizado estudio de susceptibilidad antifúngica in vitro, entre diciembre de 2004 y septiembre de 2010. La información obtenida incluyó los datos demográficos de los pacientes, procedencia, fecha del examen, tipo de muestra, levadura identificada, CIM frente a anfotericina $\mathrm{B}$, fluconazol y voriconazol y categoría de susceptibilidad. Se consideró sólo un aislado de la misma especie por paciente, registrándose dos aislados de distintas especies en 13 pacientes.

Los aislados de Candida spp., fueron identificados a nivel de especie utilizando la prueba de tubo germinal para diferenciar entre Candida albicans y no albicans y API ID 32® (bioMérieux) para determinar especie, cada vez que se trabajó una muestra.

Para el estudio de susceptibilidad antifúngica in vitro se utilizaron las categorías de interpretación habituales con respecto a susceptibilidad y resistencia. Se consideró susceptibilidad dosis dependiente (SDD) a los casos en que es posible alcanzar eficacia clínica utilizando dosis mayores que las habituales. Se utilizó el método comercial E test $^{\circledR}$, de acuerdo a las recomendaciones del fabricante. En breve, se empleó un inóculo ajustado a 0,5 McFarland (preparado a partir de solución salina estéril $(9 \%$ de $\mathrm{NaCl})$ y colonias aisladas de Candida spp., provenientes de cultivos de 24 horas en agar Sabouraud). Se usó el medio de cultivo RPMI $+2 \%$ de glucosa + MOPS tampón $+1,5 \%$ bacto agar para sembrar los aislados. Para la siembra, se realizó "doble inmersión" de la tórula haciendo torulado de la placa una vez, y luego se volvió a sumergir la tórula en la suspensión volviendo a hacer el torulado de la placa, antes de colocar las tiras con los antifúngicos. Los antifúngicos probados fueron anfotericina B $(0,002-32 \mu \mathrm{g} / \mathrm{mL})$, fluconazol $(0,016-256 \mu \mathrm{g} / \mathrm{mL})$ y voriconazol $(0,002-32$ $\mu \mathrm{g} / \mathrm{mL})$. Las cepas usadas en el control de calidad fueron C. albicans ATCC 90028 y C. parapsilosis ATCC 22019. Los puntos de corte utilizados y la categorización de los aislados en susceptible, susceptible dosis dependiente y resistente fueron los publicados por el fabricante y los contenidos en los documentos CLSI M27-A $3^{4}$ y M27-S3 5 (Tabla 1). La lectura de la susceptibilidad de los aislados a los antifúngicos probados se realizó a las 24 horas de incubación, salvo en los casos en que el desarrollo no era confluente. En el caso de anfotericina B se consideró la

Tabla 1. Puntos de corte para fármacos antifúngicos de Candida spp según criterios CLSI M27-S3 y EAS 006 (E test $\left.{ }^{\circledR}\right)$

\begin{tabular}{|c|c|c|c|}
\hline Antifúngico & $\begin{array}{l}\text { Susceptible } \\
\mu \mathrm{g} / \mathrm{mL}\end{array}$ & $\begin{array}{l}\text { Susceptible dosis dependiente } \\
\text { (SDD) } \mu \mathrm{g} / \mathrm{mL}\end{array}$ & $\begin{array}{c}\text { Resistente } \\
\mu \mathrm{g} / \mathrm{mL}\end{array}$ \\
\hline Anfotericina B & $\leq 1$ & - & $>1$ \\
\hline Fluconazol & $\leq 8$ & $16-32$ & $\geq 64$ \\
\hline Voriconazol & $\leq 1$ & 2 & $\geq 4$ \\
\hline
\end{tabular}

inhibición completa del desarrollo. En cambio, dado el fenómeno de trailing, el punto de corte para los azoles se estimó en el primer punto de inhibición significativa o de disminución de la densidad del inóculo ( $80 \%$ de inhibición del desarrollo se considera como el punto para determinar la CIM). Los resultados obtenidos por $E$ test ${ }^{\circledR}$ no fueron confirmados por microdilución.

El cálculo estadístico se realizó utilizando la prueba de $\chi^{2}$.

\section{Resultados}

Tanto a nivel intra como extra-hospitalario se registró un leve predominio de pacientes bajo 60 años de edad (52 y $61 \%$, respectivamente).

La distribución por sexo fue similar, tanto en pacientes hospitalizados como ambulatorios.

Se realizó estudio de susceptibilidad in vitro a antifúngicos a 293 aislados de Candida spp., para fluconazol y/o anfotericina B y a 257 para voriconazol.

Procedencia y tipo de muestras: 158 estudios fueron efectuados en aislados de 148 pacientes hospitalizados y 135 en aislados de 132 pacientes ambulatorios. Las especies de Candida fueron recuperadas de orina (n: 64), hemocultivos (n: 51), secreciones (n: 24), líquidos (n: 10), punta de catéter (n: 9), abscesos (n: 4), tejidos (n: 2) y 129 tenían origen desconocido por tratarse de cepas enviadas al laboratorio sólo para antifungigrama.

Especies de Candida evaluadas: C. albicans (n: 110; 38\%), C. glabrata (n: 89; 30\%), C. tropicalis (n: 33; $11 \%$ ), C. parapsilosis (n: 30; 10\%), C. krusei (n: 12; 4\%) y otras (n: 19; 7\%).

Susceptibilidad in vitro: durante el período estudiado, tanto C. albicans como Candida no albicans (C. glabrata, C. tropicalis y C. parapsilosis) presentaron $100 \%$ de sensibilidad a anfotericina B. Se observó resistencia a este antifúngico sólo en un aislado de C. krusei (1 de 12). La susceptibilidad a fluconazol se muestra en la Tabla 2. Candida albicans presentó $91,8 \%$ de sensibilidad; sin embargo, se registraron aislados con SDD (6,4\%) y resistencia $(1,8 \%)$. La especie con menor porcentaje de sensibilidad fue C. glabrata $(30,3 \%)$ y la especie $\sin$ aislados susceptibles fue C. krusei (resistencia natural). Con respecto a voriconazol, se registró resistencia en $C$. glabrata $(11,5 \%)$ y en C. parapsilosis $(3,6 \%)$.

Al considerar sólo aquellos aislados en que se probaron ambos azoles, C. albicans presentó una significativa mayor sensibilidad a fluconazol que Candida no albicans (92 vs 55\%; p <0,0001). Además, las Candida no albicans exhibieron resistencia $(6 \%)$ a voriconazol (9 C. 
Tabla 2. Susceptibilidad a antifúngicos en aislados de Candida spp

\begin{tabular}{|c|c|c|c|c|}
\hline \multirow[t]{2}{*}{ Especie de Candida } & \multirow[t]{2}{*}{ Categoría } & \multicolumn{3}{|c|}{ Antifúngicos (\%) } \\
\hline & & Anfotericina B & Fluconazol & Voriconazol \\
\hline C. albicans (n: 110) & $S$ & $110(100 \%)$ & $101(91,8 \%)$ & $96(100 \%)$ \\
\hline \multirow{2}{*}{ Voriconazol (n: 96) } & SDD & -- & $7(6,4 \%)$ & -- \\
\hline & $\mathrm{R}$ & -- & $2(1,8 \%)$ & -- \\
\hline C. glabrata $(n=89)$ & $S$ & $89(100 \%)$ & $27(30,3 \%)$ & 67 (85,9\%) \\
\hline \multirow{2}{*}{ Voriconazol (n: 78) } & SDD & -- & $35(39,3 \%)$ & $2(2,6 \%)$ \\
\hline & $\mathrm{R}$ & -- & $27(30,3 \%)$ & $9(11,5 \%)$ \\
\hline C. tropicalis (n: 33) & $S$ & $33(100 \%)$ & $31(93,9 \%)$ & 27 (100\%) \\
\hline \multirow{2}{*}{ Voriconazol (n: 27) } & SDD & -- & $1(3 \%)$ & -- \\
\hline & $\mathrm{R}$ & -- & $1(3 \%)$ & -- \\
\hline C. parapsilosis (n: 30) & $S$ & $30(100 \%)$ & $27(90 \%)$ & $27(96,4 \%)$ \\
\hline \multirow{2}{*}{ Voriconazol (n: 28) } & SDD & -- & $2(6,7 \%)$ & -- \\
\hline & $\mathrm{R}$ & -- & $1(3,3 \%)$ & $1(3,6 \%)$ \\
\hline C. krusei (n: 12) & S & $11(92 \%)$ & -- & $10(100 \%)$ \\
\hline \multirow{2}{*}{ Voriconazol (n: 10) } & SDD & -- & -- & -- \\
\hline & $\mathrm{R}$ & $1(8 \%)$ & $12(100 \%)$ & -- \\
\hline C. lusitaniae (n: 8) & S & $8(100 \%)$ & $7(88 \%)$ & $7(100 \%)$ \\
\hline \multirow{2}{*}{ Voriconazol (n: 7) } & SDD & -- & $1(12 \%)$ & -- \\
\hline & $\mathrm{R}$ & -- & -- & -- \\
\hline C. guilliermondii (n: 7) & $\mathrm{S}$ & $7(100 \%)$ & $5(71 \%)$ & $7(100 \%)$ \\
\hline \multirow[t]{2}{*}{ Voriconazol (n:7) } & SDD & -- & $2(29 \%)$ & -- \\
\hline & $\mathrm{R}$ & -- & -- & -- \\
\hline \multicolumn{5}{|l|}{ Otras: } \\
\hline \multirow{3}{*}{$\begin{array}{l}\text { Candida spp. (n: 2); } \\
\text { C. kefyr (n: 1); } \\
\text { C. sake (n: 1) }\end{array}$} & $S$ & $4(100 \%)$ & $2(100 \%)$ & $4(100 \%)$ \\
\hline & SDD & -- & 1 ** $(50 \%)$ & -- \\
\hline & $\mathrm{R}$ & -- & $1 * *(50 \%)$ & -- \\
\hline \multicolumn{5}{|l|}{ Voriconazol (n: 4) } \\
\hline $\begin{array}{l}S=\text { sensible; SDD }=\text { ser } \\
\text { je de susceptibilidad po } \\
\text { y } 1 \text { Candida spp. resiste }\end{array}$ & $\begin{array}{l}\text { ble dosis dep } \\
\text { ratarse de me } \\
\text { e a fluconaz }\end{array}$ & $\begin{array}{l}\text { endiente; } R=\text { resist } \\
\text { nos de } 30 \text { aislados; } \\
\text { l. }\end{array}$ & $\begin{array}{l}\text { nte; }{ }^{*}=\text { no se } \\
* * \\
* \text { C. sake }\end{array}$ & $\begin{array}{l}\text { Dtuvo porcenta- } \\
\text { D a fluconazo }\end{array}$ \\
\hline
\end{tabular}

Tabla 3. Susceptibilidad a azoles (\%) en aislados de Candida albicans versus Candida no albicans

\begin{tabular}{|rrrrrrr}
\hline Tipo de Candida & \multicolumn{3}{c}{ Fluconazol } & \multicolumn{3}{c}{ Voriconazol } \\
& S & SDD & R & S & SDD & R \\
C. albicans (n: 96) & 92 & 7 & 1 & 100 & - & - \\
Candida no albicans (n: 161) & 55 & 26 & 19 & 93 & 1 & 6 \\
C. glabrata (n: 78) & 33 & 41 & 26 & 86 & 3 & 11 \\
C. tropicalis (n: 27) & 92 & 4 & 4 & 100 & - & - \\
C. parapsilosis (n: 28) & 89 & 7 & 4 & 96 & - & 4 \\
C. krusei (n: 10) & - & 40 & 60 & 100 & - & - \\
C. lusitaniae (n: 7) & 86 & - & 14 & 100 & - & - \\
C. guilliermondii (n: 7) & 71 & 29 & - & 100 & - & - \\
Otras (n: 4) & 50 & 25 & 25 & 100 & - & - \\
\hline S = sensible; SDD $=$ sensible dosis dependiente; R $=$ resistente. &
\end{tabular}

glabrata y 1 C. parapsilosis). Un $45 \%$ de C. glabrata resistentes a fluconazol también fueron resistentes a voriconazol ( 9 de 20 aislados resistentes a fluconazol; Tabla 3).

En los aislados de C. albicans provenientes de sangre se observó $100 \%$ de sensibilidad a fluconazol. En los aislados de orina, la sensibilidad de C. albicans a fluconazol fue $76 \%$. La sensibilidad a voriconazol fue de $100 \%$. En el caso de Candida no albicans, se registró bajos porcentajes de sensibilidad a fluconazol, tanto en sangre como en orina (sangre, $65 \%$ y orina, 38\%) (Tabla 4). La sensibilidad a voriconazol de estos aislados fue $\geq 80 \%$ en todas las muestras.

Se comparó la sensibilidad de C. albicans y de C. glabrata (especie no albicans más frecuente en esta serie) en dos periodos de tiempo (2004-2007 vs 2008-2010), observándose que anfotericina B mantuvo excelente actividad (sensibilidad de 99\%-100\%) (datos no mostrados). C. albicans y C. glabrata no presentaron variación en su sensibilidad a fluconazol entre ambos períodos

\begin{tabular}{|c|c|c|c|c|c|c|}
\hline Tipo de Candida & Azoles & $\begin{array}{l}\text { Categoría de } \\
\text { susceptibilidad }\end{array}$ & \multicolumn{4}{|c|}{$\begin{array}{l}\text { Sitio de obtención } \\
\text { Sangre }(n=26) \quad \text { Líquidos y secreciones }(n=6)\end{array}$} \\
\hline \multirow[t]{7}{*}{ C. albicans } & \multirow[t]{3}{*}{ Fluconazol } & S & 76 & 100 & 83 & 100 \\
\hline & & SDD & 24 & 0 & 17 & 0 \\
\hline & & R & 0 & 0 & 0 & 0 \\
\hline & \multirow[t]{3}{*}{ Voriconazol } & s & 100 & 100 & 100 & 100 \\
\hline & & SDD & 0 & 0 & 0 & 0 \\
\hline & & $\mathrm{R}$ & 0 & 0 & 0 & 0 \\
\hline & & & Orina $(n=42)$ & Sangre $(n=20)$ & Líquidos y secreciones $(n=10)$ & Otra $(n=22)$ \\
\hline \multirow[t]{6}{*}{ Candida no albicans } & \multirow[t]{3}{*}{ Fluconazol } & S & 38 & 65 & 60 & 59 \\
\hline & & SDD & 36 & 20 & 10 & 27 \\
\hline & & R & 26 & 15 & 30 & 14 \\
\hline & \multirow[t]{3}{*}{ Voriconazol } & s & 90 & 95 & 90 & 86 \\
\hline & & SDD & 0 & 0 & 0 & 5 \\
\hline & & $\mathrm{R}$ & 10 & 5 & 10 & 9 \\
\hline
\end{tabular}




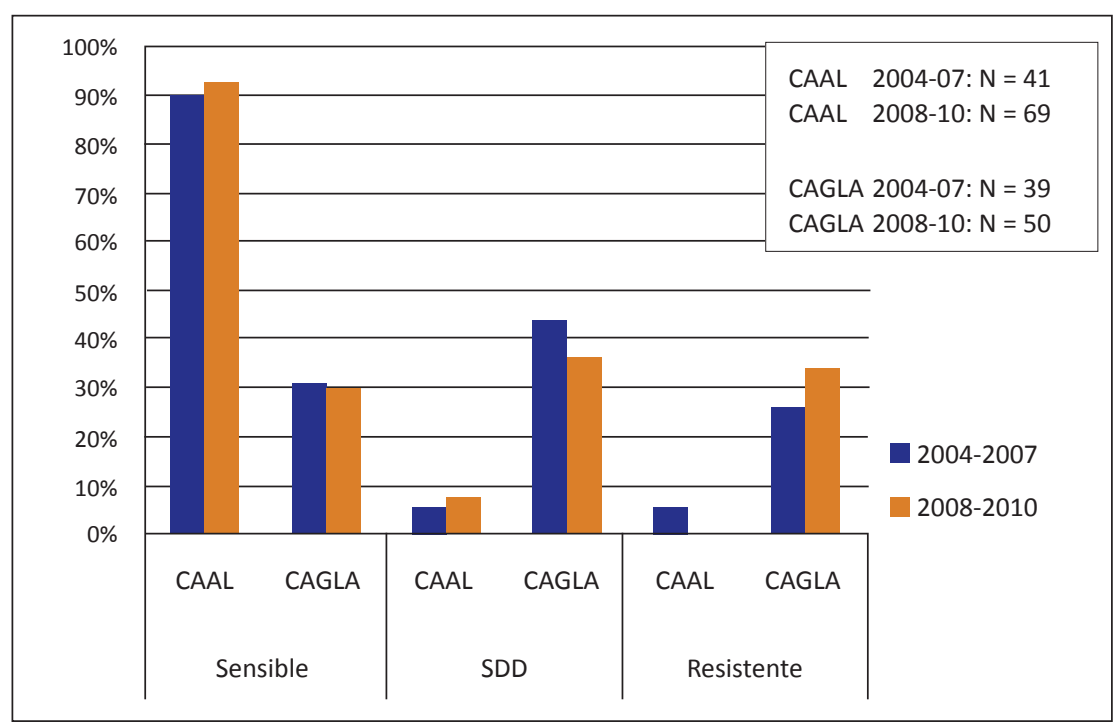

Figura 1. Susceptibilidad a fluconazol en aislados de Candida albicans (CAAL) versus Candida glabrata (CAGL) entre 2004-2007 y 2008-2010.

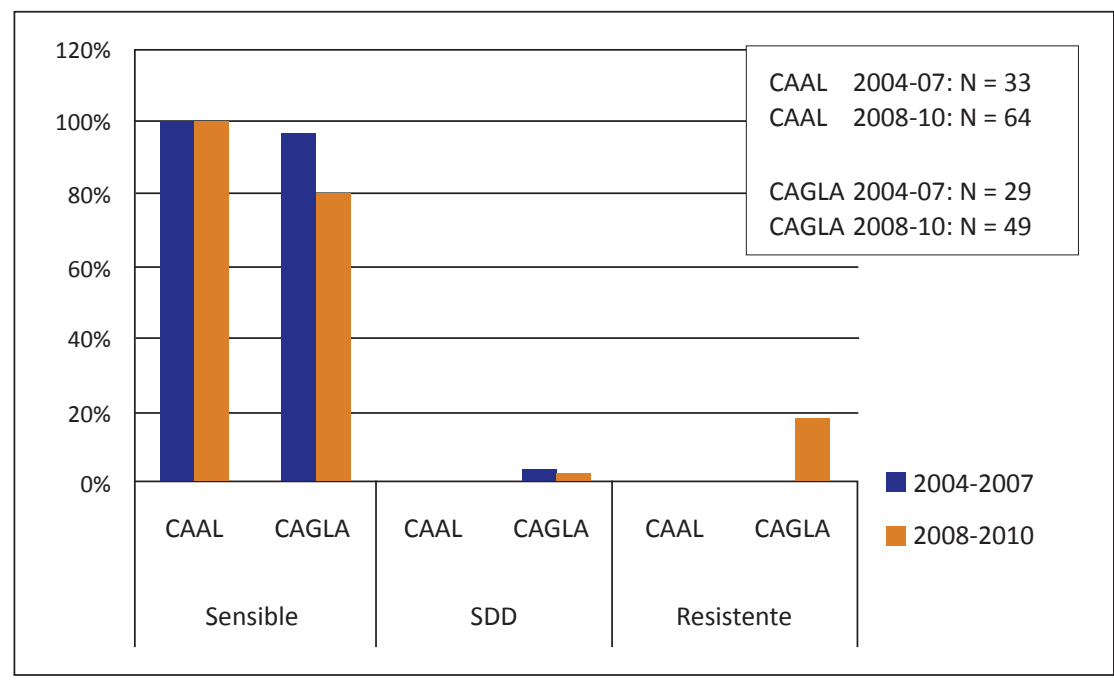

Figura 2. Susceptibilidad a voriconazol en aislados de Candida albicans (CAAL) versus Candida glabrata (CAGL) entre 2004-2007 y 2008-2010. (aproximadamente $90 \%$ en la primera y $30 \%$ en la segunda). C. glabrata experimentó la aparición de resistencia a voriconazol durante los dos últimos años estudiados, lo que representa una diferencia significativa con respecto al primer periodo (97 vs $80 \%)(\mathrm{p}<0,05)$ (Tabla 5).

\section{Discusión}

El género Candida constituyen la principal causa de infección fúngica invasora por levaduras en $\mathrm{Chile}^{7}$. $\mathrm{La}$ frecuencia de las especies responsables de infección varía según la población estudiada. En un trabajo realizado en aislados de pacientes con enfermedad fúngica invasora provenientes de cinco hospitales de la Región de Valparaíso entre 2004 y 2009, se observó que la especie más frecuente fue $C$. albicans, seguida de $C$. tropicalis y $C$. parapsilosis 7 . Sin embargo, las especies no albicans en conjunto $(57,5 \%)$ fueron más frecuentes que $C$. albicans $(42,2 \%)^{7}$. Ya en 2002 , Silva et al habían reportado $43 \%$ de C. albicans, seguido de 19\% de C. parapsilosis, $13 \%$ de C. tropicales y $3,3 \%$ de C. glabrata ${ }^{8}$. Por su parte, un estudio sobre el perfil epidemiológico de la candidiasis invasora en unidades de pacientes críticos en un hospital universitario realizado entre 2001 y 2003, reveló que del total de candidemias, $44,4 \%$ ( 8 de 18$)$ correspondieron a $C$. albicans y $55,6 \%$ a Candida no albicans (10 de18). Estos datos concuerdan con estudios chilenos, latinoamericanos y norteamericanos previos de vigilancia que han descrito un aumento de aislamientos de especies no albicans en los últimos años en infecciones fúngicas invasoras ${ }^{10}$.

Si bien nuestro estudio no permite obtener conclusiones acerca de la frecuencia de las distintas especies debido al desconocimiento de los diagnósticos de origen, los datos de los estudios chilenos recientes antes mencionados sugieren una probable distribución similar de las especies. Este perfil epidemiológico enfatiza la importancia de conocer la susceptibilidad antifúngica in vitro de los aislados, pues la resistencia a estos antifúngicos se observa predominantemente en el grupo de Candida no albicans. En el estudio de Cruz y cols., todas

Tabla 5. Susceptibilidad a azoles en aislados de Candida albicans vs Candida glabrata en dos períodos: 2004-2007 y 2008-2010

\begin{tabular}{|c|c|c|c|c|c|c|c|c|c|c|c|c|}
\hline \multirow[t]{3}{*}{ Período (años) } & \multicolumn{6}{|c|}{ C. albicans } & \multicolumn{6}{|c|}{ C. glabrata } \\
\hline & \multicolumn{3}{|c|}{ Fluconazol (n: 41) } & \multicolumn{3}{|c|}{ Voriconazol (n: 33) } & \multicolumn{3}{|c|}{ Fluconazol (n: 39) } & \multicolumn{3}{|c|}{ Voriconazol (n: 29) } \\
\hline & S & SDD & $\mathbf{R}$ & S & SDD & $\mathbf{R}$ & S & SDD & $\mathbf{R}$ & S & SDD & $\mathbf{R}$ \\
\hline \multirow[t]{2}{*}{ 2004-2007 } & $90 \%$ & $5 \%$ & $5 \%$ & $100 \%$ & - & - & $31 \%$ & $44 \%$ & $26 \%$ & $97 \%$ & $3 \%$ & - \\
\hline & \multicolumn{3}{|c|}{ Fluconazol (n: 69) } & \multicolumn{3}{|c|}{ Voriconazol (n: 64) } & \multicolumn{3}{|c|}{ Fluconazol (n: 50) } & \multicolumn{3}{|c|}{ Voriconazol (n: 49) } \\
\hline $2008-2010$ & $93 \%$ & $7 \%$ & - & $100 \%$ & - & - & $30 \%$ & $36 \%$ & $34 \%$ & $80 \%$ & $2 \%$ & $18 \%$ \\
\hline
\end{tabular}


las especies del género Candida fueron sensibles a fluconazol y a voriconazol, excepto C. glabrata ${ }^{7}$. Ajenjo et al, también documentó resistencia a fluconazol en Candida no albicans ${ }^{9}$. Alburquerque y cols., en 2007 observaron $92,3 \%$ de sensibilidad a fluconazol en C. albicans, $85,7 \%$ en C. glabrata (de las cuales, $78,6 \%$ fueron SDD) y $80 \%$ en C. tropicalis ${ }^{13}$.

Lo anterior, sumado al desarrollo de nuevos antifúngicos como las equinocandidas y azoles de amplio espectro ha complicado la elección del tratamiento antifúngico de las infecciones por Candida spp. ${ }^{14}$. Aunque C. albicans sigue siendo el patógeno más común y no presenta grandes problemas de sensibilidad antifúngica (1-2,1\% de resistencia a fluconazol), el aumento de la resistencia a azoles y frecuencia de aislamiento de especies de Candida no albicans, supone un problema al momento de decidir el esquema terapéutico más adecuado. Las especies con mayor resistencia descrita a azoles son C. krusei (resistencia intrínseca), C. glabrata (aumento de resistencia de $7 \%$ en 2001 a $12 \%$ en 2004$)^{3}$ y C. tropicalis. Las guías de la Sociedad Americana de Enfermedades Infecciosas (IDSA) sugiere que, si bien la infección invasora por C. glabrata puede tratarse con fluconazol utilizando un régimen de $\geq 12 \mathrm{mg} / \mathrm{kg} /$ día, esta terapia debe guiarse por los resultados del estudio de susceptibilidad antifúngica dada su frecuencia variable de resistencia ${ }^{15}$. También ha habido reportes aislados de resistencia a equinocandinas en pacientes con infecciones por C. albicans, C. glabrata, C. krusei y C. parapsilosis. Esta resistencia se desarrolló durante la terapia y se asoció a fracaso del tratamiento ${ }^{16-20}$.

Aunque la resistencia a anfotericina B sigue siendo excepcional, se han registrado casos de CIMs elevadas frente a este antifúngico en aislados de C. krusei y $C$. glabrata. Además, es frecuente observar resistencia intrínseca a polienos en C. lusitaniae y T. asahii ${ }^{3}$.

En nuestro medio, anfotericina B aparece como un fármaco al cual no se describe resistencia, aún en especies reconocidamente resistentes como $C$. lusitaniae. Este fenómeno se ha observado también en el extranjero ${ }^{14,21}$. En un estudio canadiense que incluyó 464 aislados, la resistencia a anfotericina fue infrecuente. Se observaron CIMs de $2 \mu \mathrm{g} / \mathrm{mL}$ o más, determinadas después de 24 horas por microdilución en caldo, en un aislado de $C$. albicans, uno de C. krusei y uno de C. lusitaniae. Sólo uno de 13 aislados de $C$. lusitaniae, presentó una CIM de $2 \mu \mathrm{g} / \mathrm{mL}$ o mayor ${ }^{15}$. Las características farmacológicas de este antifúngico han reducido su uso clínico, lo que podría explicar la escasa aparición de resistencia.

Fluconazol es un fármaco de amplio uso, tanto en el medio intra como extra-hospitalario, a pesar de su actividad generalmente fungistática. Sin embargo, su disponibilidad oral e intravenosa, su baja toxicidad y su adecuada biodisponibilidad la ponen en ventaja con respecto a anfotericina B en ciertas candidiasis ${ }^{22}$. En este contexto, destaca la alta susceptibilidad a fluconazol de C. albicans a través del tiempo $(91,8 \%)$, con sólo $6,4 \%$ de SDD y $1,8 \%$ de resistencia. Un trabajo previo realizado en Chile por Alburquerque y cols., en 2009, había demostrado la existencia de $6,8 \%$ de SDD y $0,8 \%$ de resistencia en aislados de C. albicans, recuperados fundamentalmente de flujo vagina $1^{13}$. En relación al grupo de Candida no albicans, C. glabrata fue la especie con mayor proporción de aislados en la categoría SDD, lo que concuerda con la literatura científica. Esta condición estaría asociada al carácter haploide de la levadura ${ }^{2}$, el que explicaría en parte por qué las mutaciones que llevan a la resistencia a fluconazol surgen con mayor frecuencia en este microorganismo ${ }^{23}$. La resistencia de $30,3 \%$ a fluconazol en C. glabrata demuestra un aumento progresivo en comparación al 14,3\% publicado en cepas nacionales en $2009^{13}$ y al $15 \%$ descrito en aislados de sangre durante un seguimiento de 12 años $^{24}$. La resistencia cruzada con otros azoles es común en esta especie ${ }^{25}$, lo que también fue observado en nuestros aislados (aproximadamente la mitad fue también resistente a voriconazol). La aparición de SDD a fluconazol en C. tropicalis, C. parapsilosis, C. Iusitaniae, C. guilliermondii y C. sake, encontrada en nuestro trabajo había sido descrita con anterioridad ${ }^{8}$, incluyendo Chile $^{13}$. Dado el escaso número de aislados de otras Candida no albicans incluido en esta revisión, resulta recomendable aumentar la serie antes de emitir conclusiones con respecto al comportamiento de los azoles.

En este estudio se incluyeron levaduras menos frecuentes en muestras clínicas como C. lusitaniae y C. guilliermondii. A pesar de tratarse de un número muy reducido $(8$ y 7 , respectivamente), se registraron 3 aislados con SDD a fluconazol. Esta observación concuerda con la literatura científica, pues se describe que estas especies exhiben resistencia innata o adquirida a uno o más antifúngicos ${ }^{26}$.

El análisis de la susceptibilidad antifúngica según muestra de origen de los aislados demostró 100\% de sensibilidad a fluconazol en C. albicans aisladas de sangre, lo que concuerda con los datos publicados recientemente por Ajenjo y cols., con respecto a candidemias registradas entre 2001 y 2003 en pacientes críticos en nuestro hospital universitario 9 . Dicho trabajo también destaca una alta sensibilidad a fluconazol en Candida no albicans procedentes de sangre ( $80 \% ; 8$ de 10 cepas). Si bien en ambos estudios se utilizó el método de $E$ test $^{\circledR}$ para determinar la susceptibilidad a antifúngicos, esta cifra difiere de la obtenida en el presente trabajo, donde la sensibilidad de este tipo de aislados a fluconazol fue de $65 \%$. El número de aislados de Candida no albicans obtenidos de sangre en ambos estudios fue limitado (10 y 20 , respectivamente) por lo que un mayor número de cepas permitiría comprobar estos hallazgos.

La comparación de la susceptibilidad de C. albicans y 
C. glabrata entre los periodos 2004-2007 y 2008-2010, reveló la mantención de alta sensibilidad a fluconazol en C. albicans y la aparición de la resistencia a voriconazol en C. glabrata. Este hecho constituye una alerta, sobre todo al considerar que C. glabrata presentó la segunda frecuencia en candidemias por Candida no albicans en un estudio reciente ${ }^{9}$. Además, a pesar de exhibir porcentajes de sensibilidad a anfotericina B cercanos a $100 \%$ a través del tiempo, este antifúngico no siempre constituye una alternativa viable en pacientes con patologías graves o crónicas.

Las limitaciones de este estudio son la ausencia de implicancias clínicas directas, dado que está basado en datos de laboratorio, los cuales muchas veces no incluían la información del cuadro clínico que había originado la solicitud del examen. Si bien se utilizaron puntos de corte válidos a la fecha, éstos podrían sufrir modificación en el futuro, a la luz de propuestas recientes ${ }^{27,28,29}$. Estos cambios afectarían a fluconazol, voriconazol y las equinocandinas y serían específicos según la especie de candida. La nueva información abre la posibilidad de que los datos aquí presentados requieran reevaluación en el futuro próximo. Estos resultados corresponden sólo a un centro diagnóstico, por lo que podría no estar reflejando la realidad de todo el país. Además, el método utilizado para determinar la susceptibilidad antifúngica no fue el estándar recomendado por el CLSI M27-A3 (microdilución en caldo $)^{5}$ sino el $\mathrm{E}$ test ${ }^{\circledR}$. Si bien esta metodología comercial se considera confiable y reproducible y con una correlación adecuada con el método $\mathrm{CLSI}^{3,31}$ sería recomendable efectuar un estudio prospectivo comparando los resultados simultáneos obtenidos por ambos métodos.

En virtud de los datos presentados, podemos concluir que resulta necesario realizar vigilancia de la susceptibilidad a antifúngicos en forma periódica. Anfotericina B se mantiene como un fármaco con excelente actividad in vitro frente al género Candida. Fluconazol sigue siendo una alternativa terapéutica empírica válida para C. albicans, destacando el $100 \%$ de sensibilidad encontrado en aislados provenientes de candidemias. En cambio, en Candida no albicans, es recomendable realizar estudio de susceptibilidad en aislados provenientes de muestras invasoras (sangre, líquidos o cavidades estériles), en casos seleccionados de aislados de orina y en caso de sospecha de C. glabrata. En esta especie, la resistencia a fluconazol es alta y la resistencia cruzada a voriconazol es frecuente. El aumento de resistencia a voriconazol en los últimos años requiere ser monitorizado en el tiempo y refuerza la necesidad del uso adecuado de los nuevos antifúngicos.

\section{Resumen}

Objetivo: Describir la susceptibilidad antifúngica in vitro (anfotericina B, fluconazol y voriconazol) de aislados de Candida spp., observada desde diciembre 2004 a septiembre 2010 en el Laboratorio de Microbiología de la Pontificia Universidad Católica de Chile. Material y Método: Se realizó estudio de susceptibilidad mediante $\mathrm{E}$ test $^{\circledR}$ a todas las cepas invasoras y a aquellas en que se solicitara dirigidamente antifungigrama (aislados locales o derivados desde otros centros). Resultados: Se estudiaron 293 aislados, principalmente de muestras de orina (n: 64), hemocultivos (n: 51) y secreciones (n: 24). Las especies correspondieron a C. albicans (38\%), C. glabrata (30\%), C. tropicalis $(11 \%)$, C. parapsilosis $(10 \%)$, C. krusei (4\%) y otras (7\%). Hubo $100 \%$ de susceptibilidad a anfotericina B, excepto en C. krusei (1/12 resistente). La susceptibilidad a fluconazol fue: C. albicans $91,8 \%$ (100\% en aislados de sangre vs $76 \%$ en orina); C. tropicalis, $93,9 \%$, C. parapsilosis, $90 \%$ y C. glabrata 30,3\%. Candida krusei, no tuvo aislados susceptibles a fluconazol. La resistencia a voriconazol se evidenció fundamentalmente en C. glabrata (11,5\%). Conclusiones: Recomendamos realizar estudio de susceptibilidad en aislados de muestras invasoras, casos seleccionados de cepas de orina y ante sospecha de C. glabrata. Fluconazol mantiene efectividad en C. albicans proveniente de sangre.

\section{Referencias}

1.- Pfaller M A, Diekema D J. Epidemiology of invasive candidiasis: a persistent public health problem. Clin Microbiol Rev 2007; 20: 133-63.

2.- Kiraz N, Dag I, Oz Y, Yamac M, Kiremitci A, Kasifoglu N. Correlation between broth microdilution and disk diffusion methods for antifungal susceptibility testing of caspofungin, voriconazole, amphotericin $\mathrm{B}$, itraconazole and fluconazole against Candida glabrata. J Microbiol Methods 2010; 82: 136-40.

3.- Lass-Fiörl C, Perkhofer S, Mayr A. In vitro susceptibility testing in fungi: a global perspective on a variety of methods. Mycoses
2010; 53: 1-11.

4.- CLSI. Reference method for broth dilution antifungal susceptibility testing of yeasts; approved standard-Third Edition. M27-A3 Vol. 28 No. 14, 2008.

5.- CLSI. Reference method for broth dilution antifungal susceptibility testing of yeasts; Third informational supplement. M27-S3 Vol. 28 No. 15, April 2008.

6.- Motta A L, Almeida G M, Almeida Júnior J N, Burattini M N, Rossi F. Candidemia epidemiology and susceptibility profile in the largest Brazilian teaching hospital complex. Braz J Infect Dis 2010; 14 (5): 441-8.

7.- Cruz R, Piontelli E. Enfermedad fúngica invasora en pacientes de cinco hospitales de la Región de Valparaíso, Chile. 2004 a 2009. Rev Chil Infectol 2011; 28 (2): 123-9.

8.- Silva V, Díaz MC, Febré N y Red de Diagnóstico en Micología Médica. Vigilancia de la resistencia de levaduras a antifúngicos. Rev Chil Infectol 2002; 19: 149-56.

9.- Ajenjo MC, Aquevedo S, Guzmán AM, Poggi H, Calvo M, Castillo C, et al. Perfil epidemiológico de la candidiasis invasora en unidades de pacientes críticos en un hospital universitario. Rev Chil Infectol 2011; 28(2): 118-22.

10.- Pfaller M A, Jones R N, Doern G, Sader H S, Hollis R J, Messer S A, for the Sentry 
Participant Group. International surveillance of bloodstream infections due to Candida species: frequency of occurrence and antifungal susceptibilities of isolates collected in 1997 in the United States, Canada and South America for the SENTRY Program. J Clin Microbiol 1998; 36: 1886-9.

11.- Alvarado D, Díaz M C, Silva V. Identificación y susceptibilidad antifúngica de Candida spp. aisladas de micosis invasora. Influencia del porcentaje de inhibición de crecimiento para la determinación de la CIM. Rev Med Chile 2002; 130 (4): 416-23.

12.- Silva V, Díaz M C, Febré N, and Chilean Invasive Infection Group. Invasive fungal infections in Chile: a multicenter study of fungal prevalence and susceptibility during a 1-year period. Med Mycol 2004; 42: 333-9.

13.- Alburquerque C, Hermosilla G, Tapia C. Distribución y susceptibilidad a fluconazol de levaduras del género Candida aisladas en pacientes hospitalizados y ambulatorios. Rev Chil Infectol 2009; 26 (5): 435-39.

14.- Bourgeois N, Dehandschoewercker L, Bertout S, Bousquet P, Rispail P, Lachaud L. Antifungal susceptibility of 205 Candida spp. isolated primarily during invasive candidiasis and comparison of the Vitek 2 system with the CLSI broth microdilution and $\mathrm{E}$ test methods. J Clin Microbiol 2010; 48 (1): 154-61.

15.- Pfaller M, Diekema D, Gibbs D, Newell V, Barton R, Bijie H, et al. Geographic variation in the frequency of isolation and fluconazole and voriconazole susceptibilities of Candida glabrata: an assessment from the ARTEMIS DISK global antifungal surveillance program. Diagn Microbiol Infect Dis 2010; 67: 162-71.

16.- Laverdiere M, Lalonde R G, Baril J G, Sheppard D C, Park S, Perlin D. Progressive loss of echinocandin activity following prolonged use for treatment of Candida albicans oesophagitis. J Antimicrob Chemother 2006; 57: 705-8

17.- Hernandez S, Lopez-Ribot JL, Najvar LK, McCarthy DI, Bocanegra R, Graybill JR. Caspofungin resistance in Candida albicans: correlating clinical outcome with laboratory susceptibility testing of three isogenic isolates serially obtained from a patient with progressive Candida esophagitis. Antimicrob Agents Chemother 2004; 48: 1382-3.

18.- Thompson III G, Wiederhold N, Vallor A, Villareal N, Lewis II J, Patterson T. Antimicrob Agents Chemother 2008; 52 (10): 3783-5.

19.- Hakki M, Staab J F, Marr K A. Emergence of a Candida krusei isolate with reduced susceptibility to caspofungin during therapy. Antimicrob Agents Chemother 2006; 50: 2522-4.

20.- Moudgal V, Little T, Boikov D y Vazquez J. Multiechinocandin and multiazole-resistant Candida parapsilosis isolates serially obtained during therapy for prosthetic valve endocarditis. Antimicrob Agents Chemother 2005; 49: 767-9.

21.- St-Germain G, Laverdière $M$, Pelletier R, René P, Bourgault AM, Lemieux C, et al. Epidemiology and antifungal susceptibility of bloodstream Candida isolates in Quebec: report on 453 cases between 2003 and 2005. Can J Infect Dis Med Microbiol 2008; 19 (1): 55-62.

22.- Arikan S, Rex J. Chapter 129: Antifungal agents. En Murray P, Baron E, Jorgensen J, Landry M, Pfaller M, eds. Manual of Clinical Microbiology, $9^{\text {th }}$ ed. Washington, D.C., ASM Press; 2007, p. 1949-71.

23.- Moran G, Sullivan D, Coleman D. Chapter 4: Emergence of non-Candida albicans Candida species as pathogens. En Calderone R, ed. Candida and candidiasis, Washington, D.C., ASM Press; 2002, p. 41. http://books.google.cl/, 16/01/2011.

24.- Pfaller M, Diekema D. Twelve years of fluconazole in clinical practice: global trends in species distribution and fluconazole susceptibility of bloodstream isolates of Candida. Clin Microbiol Infect 2004; 10: 11-23.

25.- Sabatelli F, Patel R, Mann P A, Mendrick C A, Norris C C, Hare R, et al. In vitro activities of posaconazole, fluconazole, itraconazole, voriconazole, and amphotericin B against a large collection of clinically important molds and yeasts. Antimicrob Agents Chemother 2006; 50 (6): 2009-15.

26.- Pfaller M, Diekema D, Mendez M, Kibbler C, Erzsebet P, Chang S-C, et al. Candida guilliermondii, an opportunistic fungal pathogen with decreased susceptibility to fluconazole: geographic and temporal trends from the ARTEMIS DISK antifungal surveillance program. J Clin Microbiol 2006; 44 (10): 3551-6.

27.- Pfaller M A, Diekema D J, Andes D, Arendrup M C, Brown S D, Lockhart S R, et al. Clinical breakpoints for the echinocandins and Candida revisited: integration of molecular, clinical, and microbiological data to arrive all species-specific interpretative criteria. Drug Resistance Updates 2011; 14: 164-76.

28.- Pfaller M A, Andes D, Diekema D J, Espinel-Ingroff A, Sheehan D. Wild-type MIC distributions, epidemiological cut-off values and species-specific clinical breakpoints for fluconazole and Candida: time for harmonization of CLSI and EUCAST broth microdilutions methods. Drug Resistance Updates 2010; 13: 180-95.

29.- Pfaller M A, Andes D, Arendrup M C, Diekema D J, Espinel-Ingroff A, Alexander B, et al. Clinical breakpoints for voriconazole and Candida spp. Revisited: review of microbiologic, molecular, pharmacodynamic, and clinical data as they pertain to the development of species-specific interpretative criteria. Diagn Microbiol Infect Dis 2011; 70: 330-43.

30.- Cuenca-Estrella M, Gómez-López A, Alastruey-Izquierdo A, Bernal-Martínez L, Cuesta I, Buitrago M, et al. Comparison of the Vitek 2 antifungal susceptibility system with the Clinical and Laboratory Standards Institute (CLSI) and European Committee on Antimicrobial Susceptibility Testing (EUCAST) broth microdilution reference methods and with the Sensititre YeastOne and E-test techniques for in vitro detection of antifungal resistance in yeast isolates. J Clin Microbiol 2010; 48 (5): 1782-6. 